\title{
Predicting Chaos with Lyapunov Exponents: Zero Plays no Role in Forecasting Chaotic Systems
}

\author{
Dominique Guégan ${ }^{1}$ and Justin Leroux ${ }^{2}$ \\ ${ }^{1}$ Université Paris1 Panthéon-Sorbonne, 106-112 boulevard de l'Hôpital, 75013 Paris \\ ${ }^{2}$ Institute for Applied Economics, HEC Montréal, CIRANO and CIRPÉE, 3000 chemin de \\ la Côte-Ste-Catherine, Montréal, QC H3T 2A7 \\ ${ }^{1}$ France \\ ${ }^{2}$ Canada
}

\section{Introduction}

When taking a deterministic approach to predicting the future of a system, the main premise is that future states can be fully inferred from the current state. Hence, deterministic systems should in principle be easy to predict. Yet, some systems can be difficult to forecast accurately: such chaotic systems are extremely sensitive to initial conditions, so that a slight deviation from a trajectory in the state space can lead to dramatic changes in future behavior.

We propose a novel methodology for forecasting deterministic systems using information on the local chaoticity of the system via the so-called local Lyapunov exponent (LLE). To the best of our knowledge, while several works exist on the forecasting of chaotic systems (see, e.g., Murray, 1993; and Doerner et al, 1991) as well as on LLEs (e.g., Abarbanel, 1992; Wolff, 1992; Eckhardt \& Yao, Bailey, 1997), none exploit the information contained in the LLE to forecasting. The general intuition behind our methodology can be viewed as a complement to existing forecasting methods, and can be extended to chaotic time series.

In this chapter, we start by illustrating the fact that chaoticity generally is not uniform on the orbit of a chaotic system, and that it may have considerable consequences in terms of the prediction accuracy of existing methods. For illustrative purposes, we describe how our methodology can be used to improve upon the well-known nearest-neighbor predictor on three deterministic systems: the Rössler, Lorenz and Chua attractors. We analyse the sensitivity of our methodology to changes in the prediction horizon and in the number of neighbors considered, and compare it to that of the nearest-neighbor predictor.

The nearest-neighbor predictor has proved to be a simple yet useful tool for forecasting chaotic systems (see Farmer \& Sidorowich, 1987). In the case of a one-neighbor predictor, it takes the observation in the past which most resembles today's state and returns that observation's successor as a predictor of tomorrow's state. The rationale behind the nearest-neighbor predictor is quite simple: given that the system is assumed to be deterministic and ergodic, one obtains a sensible prediction of the variable's future by looking back at its evolution from a similar, past situation. For predictions more than one step ahead, the procedure is iterated by successively merging the predicted values with the observed data. 
The nearest-neighbor predictor performs reasonably well in the short run (Ziehmann et al, 2000; Guégan, 2003). Nevertheless, by construction it can never produce an exact prediction because the nearest neighbor on which predictions are based can never exactly coincide with today's state-or else the underlying process, being deterministic, would also be periodic and trivially predicted. The same argument applies to other non-parametric predictors, like kernel methods, radial functions, etc. (see, e.g., Shintani \& Linton, 2004; Guégan \& Mercier, 1998). Hence, we argue that these predictors can be improved upon by correcting this inherent shortcoming.

Our methodology aims at correcting the above shortcoming by incorporating information carried by the system's LLE into the prediction. The methodology yields two possible candidates, potentially leading to significant improvements over the nearest neighbor predictor, provided one manages to solve the selection problem, which is an issue we address here. We develop a systematic method for solving the candidate selection problem and show, on three known chaotic systems, that it yields statisfactory results (close to a $100 \%$ success rate in selecting the "right" candidate).

The rest of the paper is organized as follows. In Section 2, we present our methodology on the use of LLEs in forecasting and introduce the candidate selection problem. In Section 3, we solve the selection problem and show using simulated chaotic systems that the size of the LLEs plays no role in the optimality of the selection procedure. However, the size of the LLEs does matter for the success rate of our selection algorithm and has an impact on the size of errors. These findings, as well as the sensitivity analysis of our methodology to the prediciton horizon and the number of neighbors, are presented in Section 4 . Section 5 concludes.

\section{Chaoticity depends on where you are}

Consider a one-dimensional series of $T$ observations from a chaotic system, $\left(x_{1}, \ldots x_{T}\right)$, whose future values we wish to forecast. Here, we consider that a chaotic system is characterized by the existence of an attractor in a $d$-dimensional phase space (Eckmann \& Ruelle, 1985), where $d>1$ is the embedding dimension. ${ }^{1}$ A possible embedding method involves building a $d$-dimensional orbit, $\left(X_{t}\right)$, with $X_{t}=\left(x_{t}, x_{t-\tau}, \ldots, x_{t-(d-1) \tau}\right){ }^{2}$ For the sake of exposition, we shall assume $\tau=1$ in the remainder of the paper.

By definition, the local Lyapunov exponent (LLE) of a dynamical system characterizes the rate of separation of points infinitesimally close on an orbit. Formally, two neighboring points in phase space with initial separation $\delta X_{0}$ are separated, $t$ periods later, by the distance:

$$
\delta X=\delta X_{0} e^{\lambda_{0} t}
$$

where $\lambda_{0}$ is the (largest) LLE of the system in the vicinity of the initial points. Typically, this local rate of divergence (or convergence, if $\lambda_{0}<0$ ) depends on the orientation of the initial vector $\delta X_{0}$. Thus, strictly speaking, a whole spectrum of local Lyapunov exponents exists, one per dimension of the state space. A dynamic system is considered to be (locally) chaotic if $\lambda_{0}>0$, and (locally) stable if $\lambda_{0}<0$. (see, e.g., Bailey, 1997)

We develop a methodology which exploits the local information carried by the LLE to improve upon existing methods of reconstruction and prediction. Our methodology utilizes the

\footnotetext{
${ }^{1}$ The choice of the embedding dimension has been the object of much work (see Takens, 1996, for a survey) and is beyond the scope of this work.

${ }^{2}$ Throughout the paper, capital letters will be used to denote vectors (e.g., $X$ ) while small caps letters denote real values (e.g., $x$ ).
} 
(estimated) value of the LLE to measure the intrinsic prediction error of existing predictors and corrects these predictors accordingly. Note that this methodology applies regardless of the sign of $\lambda_{i}$; i.e., regardless of whether the system is locally chaotic or locally stable. The only drawback of our approach is that it generates two candidate predictions, denoted $\hat{x}_{T}^{-}$ and $\hat{x}_{T}^{+}$, one being an excellent predictor (which improves upon existing methods) and the other being rather poor. For instance, when applied to the nearest-neighbor predictor, the candidates are the two solutions to the equation:

$$
\left(z-x_{i+1}\right)^{2}+\left(x_{T}-x_{i}\right)^{2}+\ldots+\left(x_{T-d+2}-x_{i-d+2}\right)^{2}-\left|X_{T}-X_{i}\right|^{2} e^{2 \hat{\lambda}_{i}}=0,
$$

where $X_{i}$ is the phase-space nearest neighbor of the last observation, $X_{T}$. $\lambda_{i}$ is estimated by $\hat{\lambda}_{i}$ using the method developed in Wolff (1992). ${ }^{34}$

Hence, accurate prediction boils down to being able to select the better of the two candidate predictors. Our goal here is to improve on previous work in Guégan \& Leroux (2009a, 2009b) by developing a systematic selection method to accurately select the best of the two candidates, $\hat{x}_{T}^{-}$and $\hat{x}_{T}^{+}$. To do so, we further exploit the information conveyed by the LLE. Indeed, the LLE being a measure of local chaoticity of a system (Abarbanel, 1992; Wolff, 1992), it may also yield important clues regarding the regularity of the trajectory.

In fact, even "globally chaotic" systems are typically made up of both "chaotic regions", where the LLE is positive, and more stable regions where it is negative (Bailey, 1997), as we illustrate in Figures 1, 2 and 3 for the Rössler ${ }^{5}$, the Lorenz ${ }^{6}$, and the Chua ${ }^{7}$ systems, respectively ${ }^{8}$. In each figure we display, clockwise from the upper left corner: the 3-dimensional attractor in the $(x, y, z)$-space, the value of the LLE along the orbit ( $\lambda$ is displayed on the vertical axis), the value of the LLE along the trajectory, and the distribution of LLE values ranked from highest

${ }^{3}$ Other estimations of Lyapunov exponents exist. See, e.g., Gençay (1996), Delecroix et al (1997) and Bask \& Gençay (1998).

${ }^{4}$ Details on this step of the method can be found in Guégan \& Leroux (2009a, 2009b).

${ }^{5}$ We followed the $z$ variable of the following Rössler system:

$$
\left\{\begin{array}{c}
\frac{d x}{d t}=-y-z \\
\frac{d y}{d t}=x+0.1 y \\
\frac{d z}{d t}=0.1+z(x-14)
\end{array}\right.
$$

with initial values $x_{0}=y_{0}=z_{0}=0.0001$ and a step size of 0.01 (Guégan, 2003).

${ }^{6}$ We followed the $x$ variable of the following Lorenz system:

$$
\left\{\begin{array}{c}
\frac{d x}{d t}=16(y-x) \\
\frac{d y}{d t}=x(45.92-z)-y \\
\frac{d z}{d t}=x y-4 z
\end{array}\right.
$$

with initial values $x_{0}=-10, y_{0}=-10$ and $z_{0}=30$, and a step size of 0.01 (Lorenz, 1963).

7 We followed the $z$ variable of the following Chua system:

$$
\left\{\begin{array}{c}
\frac{d x}{d t}=9.35(y-h(x)) \\
\frac{d y}{d t}=x-y+z \\
\frac{d z}{d t}=-14.286 y
\end{array},\right.
$$

with $h(x)=\frac{2}{7} x-\frac{3}{14}(|x+1|-|x-1|)$ initial values $x_{0}=0.3, y_{0}=-0.3$ and $z_{0}=0.28695$, and a step size of 0.01 . For an exhaustive gallery of double scroll attractors, see Bilotta et al (2007).

${ }^{8}$ For each attractor, we simulated 30,000 observations and deleted the first 5,000 ensure that we are working within the attractor. 
to lowest. Notice that for each attractor, the value of the LLE takes on positive and negative values (i.e., above and below the $\lambda=0$ plane depicted in the upper-right corner). Hence, we may expect very stable trajectories where the LLE is small, wheras regions where the LLE is large yield highly unstable behavior.

(a) XYZ-plot of the Rossler attractor. 5000 observations.
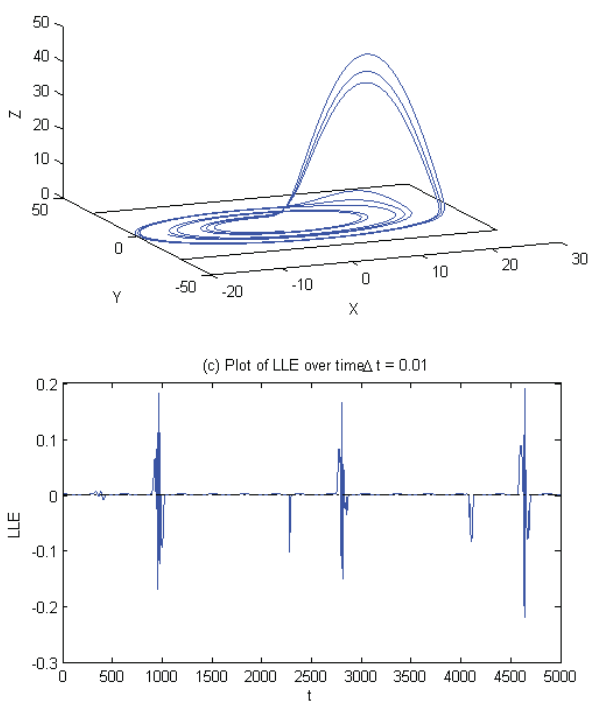

(b) Plot of LLE on the XY plane of the Rossler attractor
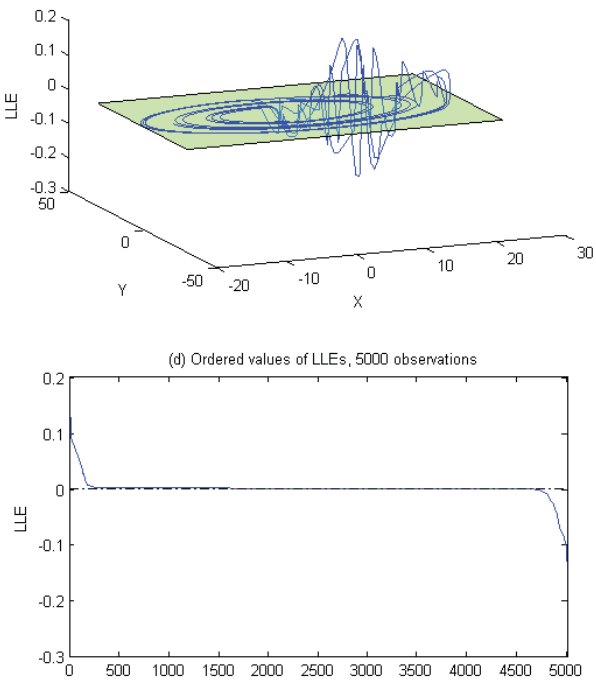

Fig. 1. Evolution of the LLE for the Rössler system

\section{Solving the selection problem}

Assuming that we observe $x_{1}, \ldots, x_{T}$, and following the insights of the previous section, we now investigate conditioning our selection process on the value of the LLE. Formally, our algorithm can be defined as follows:

$$
\left\{\begin{array}{l}
\text { If } \lambda_{T} \leq \bar{\lambda}, \text { select the "colinear" candidate } \\
\text { otherwise, select the "non colinear" candidate, }
\end{array}\right.
$$

where $\bar{\lambda}$ is an exogenously given threshold value. We abuse terminology slightly and denote by "colinear" the candidate which maximizes the following scalar product:

$$
\hat{X}_{T+1}^{c}=\arg \max _{\hat{X}_{T+1} \in C} \frac{\left(\hat{X}_{T+1}-X_{T}\right) \cdot\left(X_{i+1}-X_{T}\right)}{\left\|\hat{X}_{T+1}-X_{T}\right\| \times\left\|X_{i+1}-X_{T}\right\|}
$$

where $C=\left\{\left(\hat{x}_{T+1}^{-}, x_{T}, \ldots, x_{T-d+2}\right),\left(\hat{x}_{T+1}^{+}, x_{T}, \ldots, x_{T-d+2}\right)\right\}$ and $X_{i+1}$ is the successor of the nearest neighbor of $X_{T}$ in phase space. Likewise, we denote by $\hat{X}_{T+1}^{n c}$, and call "non colinear", the candidate which minimizes the scalar product in Expression (3).

In words, the algorithm assumes that when the value of the LLE is low, the orbit is relatively smooth, suggesting that the trajectory to be predicted behaves similarly as the nearest 

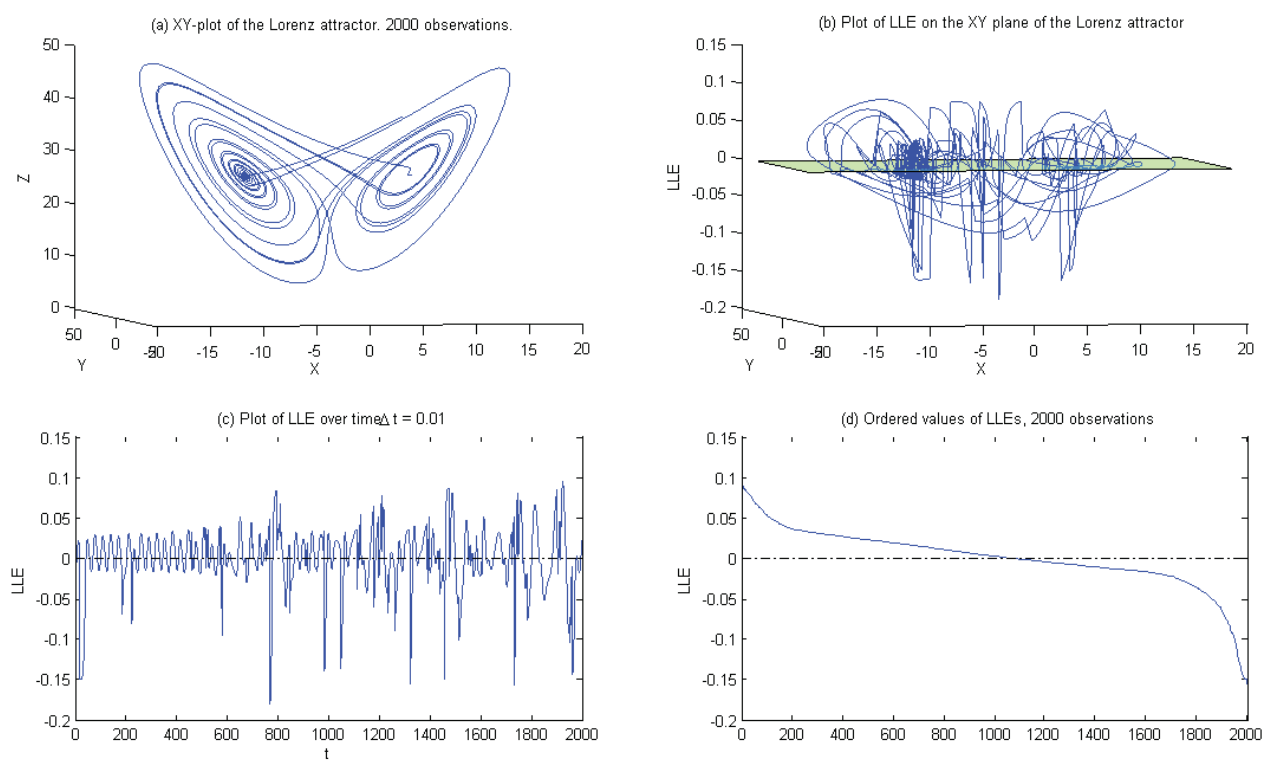

Fig. 2. Evolution of the LLE for the Lorenz system
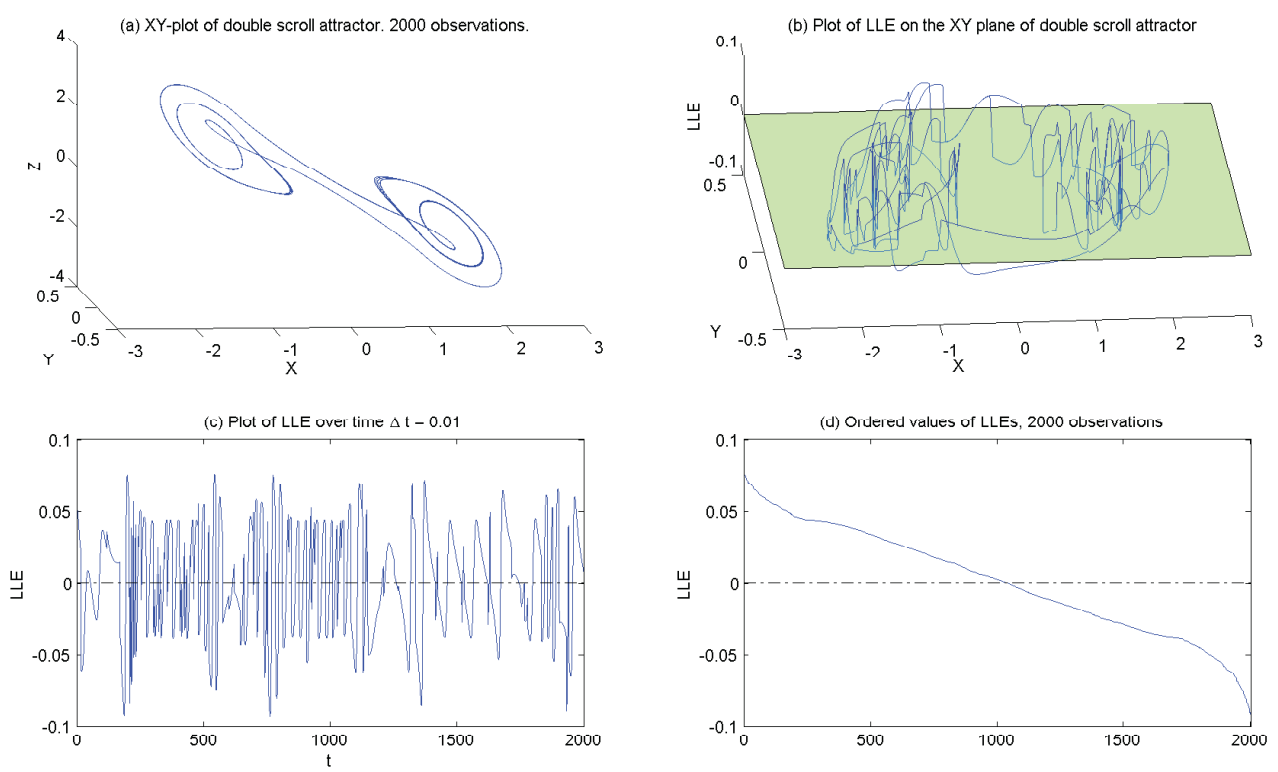

Fig. 3. Evolution of the LLE for the Chua system 
neighbor's trajectory. Alternatively, when the LLE is "large", the trajectory is considered to behave erratically, so that the trajectory to be predicted is assumed to differ from that of its nearest neighbor.

Intuition suggests that one may need to estimate the optimal value of the threshold $\bar{\lambda}$ in terms of prediction accuracy for each chaotic system. Hence, we calculate the mean squared error (MSE) of the predictor using the above selection algorithm (2) in order to assess which threshold $\bar{\lambda}$ minimizes the MSE:

$$
\operatorname{MSE}^{S}(\bar{\lambda})=\frac{1}{n} \sum_{t=T-n+1}^{T}\left(\hat{X}_{t}^{S}(\bar{\lambda})-X_{t}\right)^{2},
$$

with $\hat{X}_{t}^{s}(\bar{\lambda})=\hat{X}_{t}^{c}$ or $\hat{X}_{t}^{n c}$ according to selection algorithm (2), and where $n$ is the number of predictions. We compute $M S E^{S}(\bar{\lambda})$ across all values of $\bar{\lambda}$ in the range of the system's LLE over the last 1000 observations of our sample $(n=1000)$ using the entire, true information set leading up to the predictee for each prediction. Figure 4 plots the values of $M S E^{S}$ as a function of $\bar{\lambda}$ for the Rössler, Lorenz and Chua attractors. We find that $\operatorname{MSE}^{S}(\bar{\lambda})$ is smallest when $\bar{\lambda}$ is the upper bound of the range. In other words, our method seems to not require estimating the optimal threshold, $\bar{\lambda}$, as one is better off always selecting the colinear candidate and not conditioning the selection process on the LLE, as intuition might have suggested.
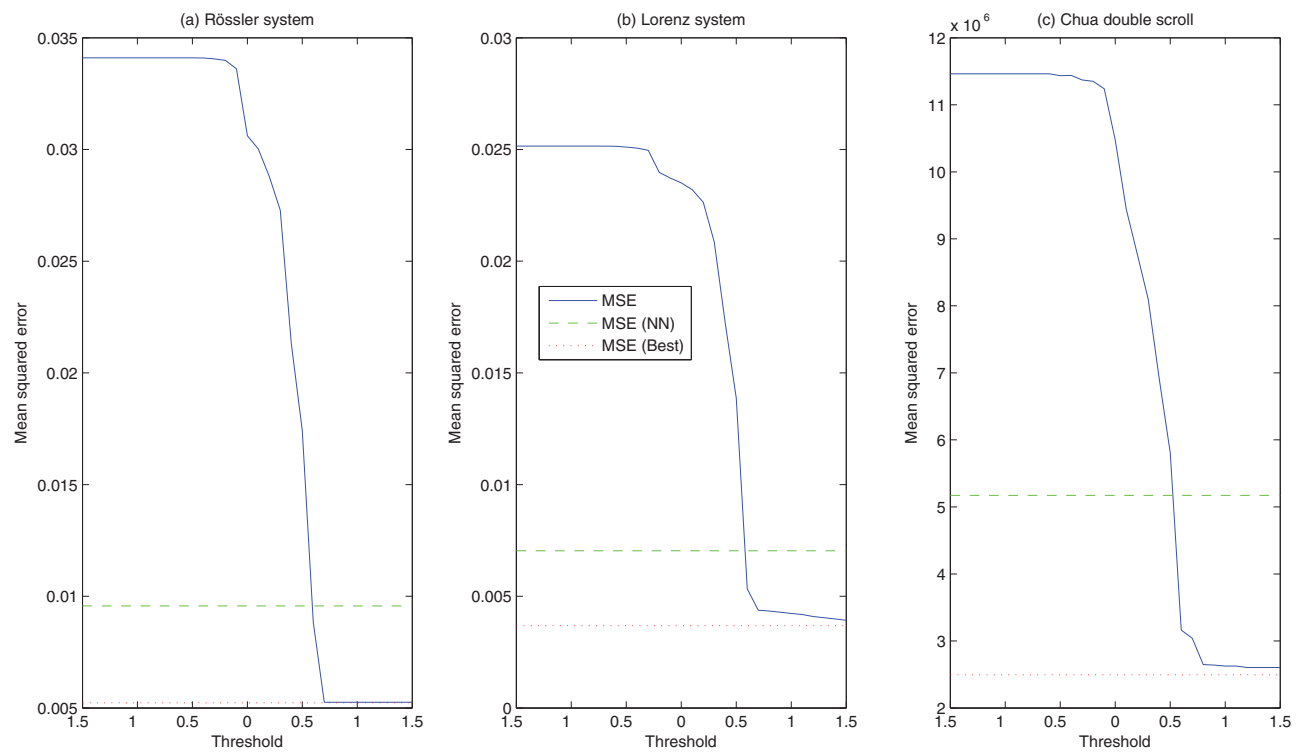

Fig. 4. MSE as a function of threshold $\bar{\lambda}$

In the remainder of the chapter, we shall focus on the performance of $\hat{X}^{c}$, the predictor which systematically selects the colinear candidate. For this predictor, the MSE writes as follows:

$$
M S E^{c}=\frac{1}{n} \sum_{t=T-n+1}^{T}\left(\hat{X}_{t}^{c}-X_{t}\right)^{2} .
$$


Table 1 displays the values of $M S E^{c}$ along with the performances of the nearest-neighbor predictor:

$$
M S E^{N N}=\frac{1}{n} \sum_{t=T-n+1}^{T}\left(\hat{X}_{t}^{N N}-X_{t}\right)^{2}
$$

and of the best of the two possible candidates

$$
M S E^{b}=\frac{1}{n} \sum_{t=T-n+1}^{T} \min _{i=c, n c}\left(\hat{X}_{t}^{i}-X_{t}\right)^{2} \cdot 9
$$

Table 1 also shows the success rate, $\rho$, in selecting the better of the two candidate as well as information on the value of the LLE on the orbit (line 6) and information on the LLE on the observations where "wrong" candidate was selected (line 7).

\begin{tabular}{|c|c|c|c|c|}
\hline \multicolumn{5}{|c|}{ Table 1: Prediction results. $n=1,000$ predictions. } \\
\hline & Rössler & Lorenz & Chua \\
\hline$M S E^{c}$ & 0.0053 & 0.0039 & $2.6038 \mathrm{e}-6$ \\
\hline$M S E^{N N}$ & 0.0156 & 0.0091 & $5.1729 \mathrm{e}-6$ \\
\hline$M S E^{b}$ & 0.0052 & 0.0037 & $2.4947 \mathrm{e}-6$ \\
\hline \multicolumn{2}{|c|}{$\rho$} & $97.3 \%$ & $94.30 \%$ & $98.7 \%$ \\
\hline$\hat{\lambda}_{t}$ & mean & 0.1302 & 0.1940 & 0.0593 \\
\cline { 2 - 5 } & $($ min;max) & $(-1.2453,0.9198)$ & $(-1.4353 ; 1.4580)$ & $(-1.0593 ; 1.1468)$ \\
\hline$\hat{\lambda}_{t \mid \text { fail }}$ & mean & 0.2582 & 0.4354 & 0.3253 \\
\cline { 2 - 5 } & (min;max) & $(-0.4824,09198)$ & $(-0.5142 ; 1.3639)$ & $(-0.5648 ; 0.5554)$ \\
\hline
\end{tabular}

Table 1. $M S E^{c}, M S E^{N N}$ and $M S E^{b}$ are as defined in (4), (5) and (6). $\rho$ is the selection success rate of the colinear selector. $\hat{\lambda}_{t}$ is the value of the LLE on the 1,000 observations to be predicted. $\hat{\lambda}_{t \mid \text { fail }}$ is the value of the LLE on the observations where the colinear selector does not select the best candidate.

For all three systems, we find that $M S E^{c}$ is substantially smaller than $M S E^{N N}$. Moreover, $M S E^{c}$ is relatively close to $M S E^{b}$, suggesting that our procedure selects the best of the two candidates quite often. In fact, on all three attractors, we obtain success rate, $\rho$, close to $100 \%$. Finally, on the few predictions where our predictor does select the "wrong" candidate, the value of the LLE is relatively high compared to the average LLE on the attractor ( 0.25 versus 0.13 for Rössler, 0.44 versus 0.19 for Lorenz, and 0.33 versus 0.06 for Chua). These findings are consistent with the intuition that prediction is more difficult in regions of the attractor which are more sensitive to initial conditions. While this finding seems to confirm that the value of the LLE plays a small role in the selection problem, recall that our results show that conditioning selection on the value of the LLE would not lead to improved predictions, as measured by $\operatorname{MSE}^{S}(\bar{\lambda})$.

\section{Forecasting}

In this section, we detail the role of the value of the LLE on the size of errors and on the performance of the selection procedure as well as the performance of the predictor in the short and medium run. 


\subsection{Role of the LLE on error size}

The following tables show the success rates of the selection procedure of $\hat{X}^{c}$ and the resulting MSE broken down in small value intervals for the LLE. Doing so allows one to assess how the performance of the procedure and of the predictor depends on the (local) chaoticity of the region considered. $\rho$ represents the ratio of the number of times the best candidate was selected over the number of predictions in the interval considered. These predictions are then broken down into the number of good selection $\left(n_{\text {succ }}\right)$ and the number of failures to select the best candidate $\left(n_{\text {fail }}\right)$. Next, $M S E^{c}$ shows the mean squared error of our predictor (using colinear selection) on each interval. $M S E^{c} \mid s u c c$. and $M S E^{c} \mid$ fail show the value of $M S E^{c}$ considering only the predictions where the best candidate was correctly and incorrectly selected, respectively. Finally, $M S E^{N N}$ displays the mean squared error of the nearest neighbor predictor on the relevant interval.

\begin{tabular}{|c|c|c|c|c|c|c|c|}
\hline \multicolumn{7}{|c|}{ Table 2: Rössler attractor, $n=1000$ predictions } \\
\hline$\hat{\lambda}_{t}$ range & $\rho$ & $n_{\text {succ }}$ & $n_{\text {fail }}$ & $M S E^{c}$ & $M S E^{\mathcal{C}} \mid$ succ & $M^{\mathrm{C}} E^{\mathrm{C}} \mid$ fail & $M_{S E^{N N}}$ \\
\hline$[-1.3,-1.1]$ & 1 & 1 & 0 & $3.91 \mathrm{e}-11$ & $3.91 \mathrm{e}-11$ & - & $3.91 \mathrm{e}-11$ \\
\hline$[-1.1,-0.9]$ & - & - & - & - & - & - & - \\
\hline$[-0.9,-0.7]$ & 1 & 5 & 0 & $1.32 \mathrm{e}-6$ & $1.32 \mathrm{e}-6$ & - & $1.34 \mathrm{e}-6$ \\
\hline$[-0.7,-0.5]$ & 1 & 68 & 0 & 0.0073 & 0.0073 & - & 0.0073 \\
\hline$[-0.5,-0.3]$ & 0.98 & 106 & 2 & 0.0033 & 0.0033 & $2.096 \mathrm{e}-5$ & 0.0034 \\
\hline$[-0.3,-0.1]$ & 0.97 & 105 & 3 & 0.0059 & 0.0060 & 0.0001 & 0.0072 \\
\hline$[-0.1,0.1]$ & 0.98 & 125 & 3 & 0.0089 & 0.0091 & 0.0000 & 0.0176 \\
\hline$[0.1,0.3]$ & 0.97 & 149 & 4 & 0.0019 & 0.0019 & 0.0009 & 0.0054 \\
\hline$[0.3,0.5]$ & 0.97 & 222 & 8 & 0.0059 & 0.0056 & 0.0132 & 0.0101 \\
\hline$[0.5,0.7]$ & 0.97 & 192 & 6 & 0.0051 & 0.0052 & 0.0009 & 0.0127 \\
\hline$[0.7,0.9]$ & - & - & - & - & - & - & - \\
\hline$[0.9,1.1]$ & 0 & 0 & 1 & $9.34 \mathrm{e}-10$ & - & $9.34 \mathrm{e}-10$ & $2.79 \mathrm{e}-11$ \\
\hline
\end{tabular}

Table 2. Each row relates to observations $X_{t}$ for which the LLE belongs to $\hat{\lambda}_{t}$ range. $\rho$ is the selection success ratio $(1=100 \%) . n_{\text {succ }}$ and $n_{\text {fail }}$ are the number of predictions for which the colinear selector selects correctly and incorrectly, respectively. $M S E^{c}$ and $M S E^{N N}$ are as defined in (4) and (5). $M S E^{\mathcal{C}} \mid S U C c$ and $M S E^{\mathcal{C}} \mid$ fail correspond to $M S E^{\mathcal{C}}$ restricted to the previously defined $n_{\text {succ }}$ and $n_{\text {fail }}$ observations, respectively.

Notice that for all three attractors the size of errors is relatively stable over the range of LLEs when selection is successful. This indicates that our method accurately corrects for the dispersion of neighboring trajectories as measured by the value of the LLE. If this were not the case, one would expect the MSE to increase monotonically with the value of LLE. In fact, errors become large only for values of the LLE near the upper end of their range (above 0.9 for the Rössler attractor, above 1.1 for the Lorenz attractor, and above 0.5 for the Chua attractor). A possible reason for this sudden increase may be that our estimator for the value of the LLEs is not sufficiently robust in regions of high chaoticity. We expect that a more sophisticated estimation method for the LLE may solve this issue, which we address in a companion paper. Notice that for the Rössler attractor, for most values of the LLE, the size of errors when failing to select is on average less than when selecting accurately. For example, for $\hat{\lambda} \in[0.5,0.7]$, 


\begin{tabular}{|c|c|c|c|c|c|c|c|c|}
\hline \multicolumn{7}{|c|}{ Table 3: Lorenz attractor, $n=1000$ predictions } \\
\hline$\hat{\lambda}_{t}$ range & $\rho$ & $n_{\text {succ. }}$ & $n_{\text {fail }}$ & $M S E^{c}$ & $M S E^{\mathcal{C}} \mid$ succ. & $M S E^{\mathcal{C}} \mid$ fail. & $M S E^{N N}$ \\
\hline$[-1.5,-1.3]$ & 1 & 1 & 0 & 0.0001 & 0.0001 & - & 0.0001 \\
\hline$[-1.3,-1.1]$ & - & - & - & - & - & - & - \\
\hline$[-1.1,-0.9]$ & 1 & 3 & 0 & 0.0016 & 0.0016 & - & 0.0016 \\
\hline$[-0.9,-0.7]$ & 1 & 3 & 0 & 0.0013 & 0.0013 & - & 0.0013 \\
\hline$[-0.7,-0.5]$ & 0.99 & 67 & 1 & 0.0033 & 0.0034 & 0.0003 & 0.0035 \\
\hline$[-0.5,-0.3]$ & 0.99 & 92 & 1 & 0.0049 & 0.0049 & 0.0000 & 0.0054 \\
\hline$[-0.3,-0.1]$ & 0.98 & 98 & 2 & 0.0056 & 0.0054 & 0.014 & 0.0098 \\
\hline$[-0.1,0.1]$ & 0.93 & 108 & 8 & 0.0038 & 0.0039 & 0.0026 & 0.0052 \\
\hline$[0.1,0.3]$ & 0.94 & 109 & 7 & 0.0041 & 0.0036 & 0.011 & 0.0077 \\
\hline$[0.3,0.5]$ & 0.96 & 195 & 8 & 0.0021 & 0.0020 & 0.0049 & 0.0088 \\
\hline$[0.5,0.7]$ & 0.91 & 223 & 22 & 0.0044 & 0.0038 & 0.0102 & 0.0079 \\
\hline$[0.7,0.9]$ & 0.90 & 18 & 2 & 0.0011 & 0.0008 & 0.0033 & 0.0012 \\
\hline$[0.9,1.1]$ & 0.81 & 13 & 3 & 0.0006 & 0.0003 & 0.0016 & 0.0016 \\
\hline$[1.1,1.3]$ & 0.82 & 9 & 2 & 0.0034 & 0.0031 & 0.0047 & 0.0027 \\
\hline$[1.3,1.5]$ & 0.80 & 4 & 1 & 0.042 & 0.052 & 0.0019 & 0.0015 \\
\hline
\end{tabular}

Table 3. Each row relates to observations $X_{t}$ for which the LLE belongs to $\hat{\lambda}_{t}$ range. $\rho$ is the selection success ratio $(1=100 \%) . n_{\text {succ }}$ and $n_{\text {fail }}$ are the number of predictions for which the colinear selector selects correctly and incorrectly, respectively. $M S E^{c}$ and $M S E^{N N}$ are as defined in (4) and (5). $M S E^{c} \mid s u c c$ and $M S E^{c} \mid$ fail correspond to $M S E^{\mathcal{C}}$ restricted to the previously defined $n_{\text {succ }}$ and $n_{\text {fail }}$ observations, respectively.

$\left.M S E^{c}\right|_{\text {succ }}=0.0052>0.0009=\left.M S E^{c}\right|_{\text {fail }}$. This apparently surprising observation is actually encouraging as it indicates that selection mistakes occur mostly when there is little need for correction. Such situations may arise because $X_{T}$ 's nearest neighbor is very close to $X_{T}$ or, alternatively, when both candidates, $\hat{x}_{T+1}^{-}$and $\hat{x}_{T+1}^{+}$are both very close to $x_{i+1}$ due to space orientation considerations. The same phenomenon can be observed for the Lorenz system up to $\hat{\lambda}=0.1$ and for $\hat{\lambda}>1.3$, but is less systematic for the Chua system.

Regarding the selection accuracy, as measured by $\rho$, we find that our algorithm selects almost perfectly for all three attractors, and in most ranges of $\hat{\lambda}$. As expected, $\rho$ dips slightly for larger values of $\hat{\lambda}$ in the case of the Rössler and Lorenz attractors, which is in line with the common intuition according to which trajectories are more stable, or smoother, where the value of the LLE is small and more irregular for large values of the LLE. Surprisingly, the Chua attractor behaves somewhat differently. Interestingly, selection mistakes occur on all attractors for negative values of the LLE, where the system is supposedly locally "stable". Hence, our results suggest that the focal value of $\lambda=0$, traditionally separating order from chaos, bears little meaning in terms of forecasting. 


\begin{tabular}{|c|c|c|c|c|c|c|c|}
\hline \multicolumn{7}{|c|}{ Table 4: Chua attractor, $n=1000$ predictions } \\
\hline$\hat{\lambda}_{t}$ range & $\rho$ & $n_{\text {succ }}$ & $n_{\text {fail }} \cdot$ & $\begin{array}{c}M S E^{c} \\
\left(\times 10^{-4}\right)\end{array}$ & $\begin{array}{c}M S E^{\mathcal{C}} \mid \text { succ. } \\
\left(\times 10^{-4}\right)\end{array}$ & $\begin{array}{c}M S E^{\mathcal{C}} \mid \text { fail. } \\
\left(\times 10^{-4}\right)\end{array}$ & $\begin{array}{c}M S E^{N N} \\
\left(\times 10^{-4}\right)\end{array}$ \\
\hline$[-1.3,-1.1]$ & 1 & 1 & 0 & 0.3111 & 0.3111 & - & .3111 \\
\hline$[-1.1,-0.9]$ & 1 & 1 & 0 & 0.1765 & 0.1765 & - & 0.1765 \\
\hline$[-0.9,-0.7]$ & - & 0 & 0 & - & - & - & - \\
\hline$[-0.7,-0.5]$ & 0.9873 & 78 & 1 & 0.0376 & 0.0381 & 0.0002 & 0.0391 \\
\hline$[-0.5,-0.3]$ & 0.98 & 98 & 2 & 0.0339 & 0.0332 & 0.0686 & 0.0362 \\
\hline$[-0.3,-0.1]$ & 1 & 116 & 0 & 0.0218 & 0.0218 & - & 0.0244 \\
\hline$[-0.1,0.1]$ & 0.9918 & 241 & 2 & 0.0074 & 0.0072 & 0.0285 & 0.0241 \\
\hline$[0.1,0.3]$ & 0.9917 & 120 & 1 & 0.0097 & 0.0097 & 0.0124 & 0.0228 \\
\hline$[0.3,0.5]$ & 0.9884 & 171 & 2 & 0.0199 & 0.0199 & 0.0183 & 0.0221 \\
\hline$[0.5,0.7]$ & 0.9740 & 150 & 4 & 0.0553 & 0.0508 & 0.2261 & 0.0239 \\
\hline$[0.7,0.9]$ & 0.8750 & 7 & 1 & 0.1333 & 0.0721 & 0.5619 & 0.0981 \\
\hline$[0.9,1.1]$ & 1 & 2 & 0 & 0.0884 & 0.0884 & - & 0.0025 \\
\hline$[1.1,1.3]$ & 1 & 2 & 0 & 0.2440 & 0.2440 & - & 0.0091 \\
\hline
\end{tabular}

Table 4. Each row relates to observations $X_{t}$ for which the LLE belongs to $\hat{\lambda}_{t}$ range. $\rho$ is the selection success ratio $(1=100 \%) . n_{\text {succ }}$ and $n_{\text {fail }}$ are the number of predictions for which the colinear selector selects correctly and incorrectly, respectively. $M S E^{c}$ and $M S E^{N N}$ are as defined in (4) and (5). $M S E^{C} \mid S U C c$ and $M S E^{C} \mid$ fail correspond to $M S E^{C}$ restricted to the previously defined $n_{\text {succ }}$ and $n_{\text {fail }}$ observations, respectively.

\subsection{Forecasting several steps ahead}

We now explore the possibility of forecasting a chaotic time series several steps ahead using our correction method. In order to make predictions $h$-steps ahead, we proceed iteratively, including the successive one-step predictions. ${ }^{10}$

In addition to extending our predictions to several steps ahead, we jointly investigate the role of the number of neighbors to consider in the prediction and in the estimation of the LLE. We estimated the LLE using Wolff's (1992) algorithm with infinite bandwith and $k$ neighbors, and applied our correction method to the average of the images of these neighbors $(k-\mathrm{NNP})$.

\subsubsection{Rössler attractor}

The following table shows $M S E^{c}$ and $M S E^{N N}$ as a function of the number of neighbors and the prediction horizon in the top and bottom half of the table, respectively. For each column, and for each predictor, the numbers shown in bold are the smallest mean squared error for each horizon. Therefore, the corresponding number of neighbors, $k$, is optimal for that horizon.

As expected, predictions are more accurate in the shorter run. Moreover, increasing the number of neighbors, $k$, generally seems to decrease the accuracy of the prediction. Note

\footnotetext{
${ }^{10}$ For instance, $\hat{X}_{t+2}$ is obtained by constructing the (estimated) history $\left(X_{1}, \ldots, X_{t}, \hat{X}_{t+1}\right)$. Next, $\hat{X}_{t+3}$ is obtained via history $\left(X_{1}, \ldots, X_{t}, \hat{X}_{t+1}, \hat{X}_{t+2}\right)$, and so on. Hence,no further information is injected to the true information set $\left(X_{1}, \ldots, X_{t}\right)$.
} 


\begin{tabular}{|c|c|c|}
\hline & \multicolumn{2}{|c|}{ Rössler attractor } \\
\hline & $h=1 \quad h=2 h=6$ & $h=7 h=10$ \\
\hline$k=1$ & $0.0053 \quad 0.01170 .1889$ & 0.31070 .8575 \\
\hline$k=2$ & $\mathbf{0 . 0 0 4 5} 0.01440 .2901$ & 0.48901 .6762 \\
\hline$k=3$ & $\begin{array}{llll}0.0058 & 0.0184 & 0.2114\end{array}$ & $0.3212 \mathbf{0 . 8 5 0 1}$ \\
\hline$k=4$ & $\begin{array}{lll}0.0077 & 0.0240 & 0.3074\end{array}$ & 0.43011 .3332 \\
\hline$k=5$ & $\begin{array}{lll}0.0091 & 0.0278 & 0.3650\end{array}$ & 0.51931 .1830 \\
\hline$k=10$ & $\begin{array}{lll}0.0103 & 0.04120 .6380\end{array}$ & 0.92282 .2703 \\
\hline$k=20$ & $\begin{array}{llll}0.0283 & 0.1178 & 1.8714\end{array}$ & 2.76816 .4980 \\
\hline$\overline{M S E^{1-N N}}$ & $\begin{array}{llll}0.0156 & 0.0315 & 0.2392\end{array}$ & 0.34020 .7928 \\
\hline$M S E^{2-N N}$ & $0.01940 .0384 \quad \mathbf{0 . 2 2 2 9}$ & 0.30170 .6318 \\
\hline$M S E^{3-N N}$ & $\begin{array}{llll}0.0228 & 0.0485 & 0.2784\end{array}$ & 0.37100 .7410 \\
\hline$M S E^{4-N N}$ & 0.02420 .05600 .3513 & 0.47110 .9528 \\
\hline$M S E^{5-N N}$ & 0.02950 .06840 .4224 & 0.56231 .1133 \\
\hline$M S E^{10-N N}$ & 0.05000 .13060 .9188 & 1.22282 .3539 \\
\hline$M S E^{20-N N}$ & 0.12470 .32822 .2649 & 2.97755 .4714 \\
\hline
\end{tabular}

Table 5. The top and bottom half of the table display $M S E^{c}$ and $M S E^{N N}$ as a function of the number of neighbors $k$ and the prediction horizon, $h$, respectively.

that this is also true for the uncorrected nearest-neighbor predictor. Finally, our correction method improves upon the uncorrected nearest-neighbor predictor up until six steps ahead.

\subsubsection{Lorenz attractor}

Here also, predictions are more accurate in the shorter run. However, unlike for the Rössler attractor, the simulation results suggest that accuracy increases with $k$ up to a point $(k=4)$. Beyond that, increasing the number of neighbors is detrimental to the accuracy of the method (except for $h=20$, which is too large a horizon for our predictions to be trusted).

As is the case with the Rössler attractor, our method performs uniformly better than the corresponding uncorrected nearest-neighbor predictor for horizons of up to seven steps ahead.

\subsubsection{Double scroll attractor}

Again, we see that our prediction results improve upon those of the corresponding uncorrected $k$-nearest-neighbor predictor, but only in the very short run (up to $h=2$ ). Also, as was the case with the other systems, the optimal number of neighbors is low: $k=2$. Beyond that number, any information carried by neighbors farther away seems to only pollute the prediction results

\section{Concluding comments}

We further developed the methodology on using the information contained in the LLE to improve forecasts. Our contributions is threefold. First, the selection problem is not an issue, and does not require conditioning candidate selection on the value of the LLE. Next, our 


\begin{tabular}{|c|c|c|}
\hline & \multicolumn{2}{|c|}{ Lorenz attractor } \\
\hline & $h=1 \quad h=2 \quad h=7$ & $h=8 h=10$ \\
\hline$k=1$ & 0.00390 .01760 .6821 & 1.03061 .9406 \\
\hline$k=2$ & $\begin{array}{lll}0.0024 & 0.0102 & 0.4151\end{array}$ & 0.62491 .2429 \\
\hline$k=3$ & $\begin{array}{lll}0.0020 & 0.0081 & 0.3387\end{array}$ & 0.51030 .9955 \\
\hline$k=4$ & $\begin{array}{llll}0.0014 & 0.0057 & 0.2873\end{array}$ & $\begin{array}{lll}0.4347 & 0.8803\end{array}$ \\
\hline$k=5$ & 0.00140 .00610 .3179 & 0.48520 .9724 \\
\hline$k=10$ & 0.00160 .00710 .3374 & 051241.0329 \\
\hline$k=20$ & 0.00210 .01010 .4322 & 0.64741 .2333 \\
\hline$M S E^{1-N N}$ & 0.00910 .02460 .3485 & 0.48770 .8730 \\
\hline$M S E^{2-N N}$ & 0.00840 .02260 .2994 & 0.41520 .7318 \\
\hline$M S E^{3-N N}$ & $\begin{array}{lll}0.0081 & 0.0220 & 0.2951\end{array}$ & 0.4087 0.7181 \\
\hline$M S E^{4-N N}$ & 0.00860 .02310 .2974 & 0.40960 .7133 \\
\hline$M S E^{5-N N}$ & 0.00910 .02430 .2991 & $0.4104 \quad 0.7123$ \\
\hline$M S E^{10-N N}$ & 0.01290 .03490 .3775 & 0.50010 .8136 \\
\hline$M S E^{20-N N}$ & 0.02070 .05620 .5397 & 0.68931 .0423 \\
\hline
\end{tabular}

Table 6. The top and bottom half of the table display $M S E^{c}$ and $M S E^{N N}$ as a function of the number of neighbors $k$ and the prediction horizon, $h$, respectively.

\begin{tabular}{ccc||ccc}
\multicolumn{5}{c}{ Chua double scroll } \\
\multicolumn{7}{c}{$h=1$} & $h=2$ & $h=3$ & $h=5$ & $h=10$ \\
$k=1$ & $2.6038 \mathrm{e}-6$ & $1.1247 \mathrm{e}-5$ & $3.2935 \mathrm{e}-5$ & $1.3694 \mathrm{e}-4$ & 0.0012 \\
$k=2$ & $1.6569 \mathrm{e}-6$ & $5.5148 \mathrm{e}-6$ & $1.5541 \mathrm{e}-5$ & $6.1758 \mathrm{e}-5$ & $5.5566 \mathrm{e}-4$ \\
$k=3$ & $\mathbf{1 . 5 3 4 4 \mathrm { e } - 6}$ & $5.6257 \mathrm{e}-6$ & $1.5912 \mathrm{e}-5$ & $6.3038 \mathrm{e}-5$ & $6.1618 \mathrm{e}-4$ \\
$k=4$ & $2.0762 \mathrm{e}-6$ & $6.9228 \mathrm{e}-6$ & $1.9519 \mathrm{e}-5$ & $7.4392 \mathrm{e}-5$ & $6.7625 \mathrm{e}-4$ \\
$k=5$ & $2.6426 \mathrm{e}-6$ & $8.7472 \mathrm{e}-6$ & $2.3965 \mathrm{e}-5$ & $8.7017 \mathrm{e}-5$ & $6.6244 \mathrm{e}-4$ \\
$k=10$ & $4.4688 \mathrm{e}-6$ & $1.7896 \mathrm{e}-5$ & $5.2198 \mathrm{e}-5$ & $1.9949 \mathrm{e}-4$ & 0.0014 \\
$k=20$ & $6.4272 \mathrm{e}-6$ & $2.7342 \mathrm{e}-5$ & $9.3183 \mathrm{e}-5$ & $4.4513 \mathrm{e}-4$ & 0.0042 \\
\hline \hline$M S E^{1-N N}$ & $5.1729 \mathrm{e}-6$ & $8.7554 \mathrm{e}-6$ & $1.6178 \mathrm{e}-5$ & $5.2720 \mathrm{e}-5$ & $4.8311 \mathrm{e}-4$ \\
$M S E^{2-N N}$ & $4.3528 \mathrm{e}-6$ & $7.9723 \mathrm{e}-6$ & $1.5174 \mathrm{e}-5$ & $4.9276 \mathrm{e}-5$ & $4.3521 \mathrm{e}-4$ \\
$M S E^{3-N N}$ & $5.9985 \mathrm{e}-6$ & $1.1757 \mathrm{e}-5$ & $2.2003 \mathrm{e}-5$ & $6.4616 \mathrm{e}-5$ & $4.7283 \mathrm{e}-4$ \\
$M S E^{4-N N}$ & $8.6114 \mathrm{e}-6$ & $1.7168 \mathrm{e}-5$ & $3.1539 \mathrm{e}-5$ & $8.6965 \mathrm{e}-5$ & $5.6469 \mathrm{e}-4$ \\
$M S E^{5-N N}$ & $1.1190 \mathrm{e}-5$ & $2.3201 \mathrm{e}-5$ & $4.2647 \mathrm{e}-5$ & $1.1362 \mathrm{e}-4$ & $6.7550 \mathrm{e}-4$ \\
$M S E^{10-N N}$ & $1.7453 \mathrm{e}-5$ & $4.5731 \mathrm{e}-5$ & $9.4048 \mathrm{e}-5$ & $2.6532 \mathrm{e}-4$ & 0.0014 \\
$M S E^{20-N N}$ & $5.5861 \mathrm{e}-5$ & $1.6005 \mathrm{e}-4$ & $3.3975 \mathrm{e}-4$ & $9.4208 \mathrm{e}-4$ & 0.0042
\end{tabular}

Table 7. The top and bottom half of the table display $M S E^{c}$ and $M S E^{N N}$ as a function of the number of neighbors $k$ and the prediction horizon, $h$, respectively. 
results confirm that it is indeed possible to use information on the LLE to improve forecasts. We also highlight an interesting fact: the focal value of $\lambda=0$, which traditionally separates order from chaos, does not play any role in the forecasting of chaotic systems. In other words, our methodology performs equally well on both stable and chaotic regions of the attractors studies. Finally, we examined the sensitivity of our methodology to varying the number $k$ of neighbors as well as of the step-ahead horizon, $h$. While our goal was not to determine the optimal number of neighbors to consider for forecasting, it seems that each attractor admits a rather low optimal number of neighbors. We have worked with a fixed embedding dimension, $d$, throughout. Now that we have ascertained the validity of the approach, the next step is to confirm its performance on real physical or financial data.

\section{References}

[1] Abarbanel, H.D.I. (1992), Local and global Lyapunov exponents on a strange attractor. in Casdagli M and Eubank S Eds, Addison-Wesley, Nonlinear Modeling and Forecasting, SFI Studies in the Science of Complexity, Proc. Vol. XII: 229-247.

[2] B. Bailey, Local Lyapunov exponents: predictability depends on where you are. Nonlinear Dynamics and Economics, Kirman et al. Eds, 1997.

[3] Bask, M. \& Gençay, R (1998). Testing chaotic dynamics via Lyapunov exponents, Physica $D, 114: 1-2$.

[4] Bilotta, E., Di Blasi, G., Stranges, F. \& Pantano, P. (2007). A Gallery of Chua Atractors. Part VI. International Journal of Bifurcation and Chaos, 17: 1801-1910.

[5] Delecroix, M., Guégan, D. \& Léorat, G. (1997) Determining Lyapunov exponents in deterministic dynamical systems, Computational Statistics and Data Analysis, 12: 93-107.

[6] Doerner, R., Hübinger, B. \& Martienssen, W., Grossman, S. \& Thomae, S. (1991). Predictability Portraits for Chaotic Motions, Chaos, Solitons and Fractals, 1: 553-571.

[7] Eckhardt, B. \& Yao, D. (1993) Local Lyapunov exponents in chaotic systems, Physica D, 65: 100-108.

[8] Eckmann, J.P. \& Ruelle, D (1985) Ergodic theory of chaos and strange attractors. Review of Modern Physics, 57: 615-656.

[9] Farmer, J.D. \& Sidorowich, J.J. (1987) Predicting chaotic time series. Physical Review Letters, 59: $845-848$.

[10] Gencay, R. (1996). A statistical framework for testing chaotic dynamics via Lyapunov exponents, Physica D, 89: 261-266.

[11] Guégan, D. (2003). Les Chaos en Finance: Approche Statistique, Economica Eds., Paris.

[12] Guégan, D. \& Leroux, J. (2009b). Forecasting chaotic systems: The role of local Lyapunov exponents, Chaos, Solitons and Fractals, 41: 2401-2404.

[13] Guégan, D. \& Leroux, J. (2009a). Local Lyapunov Exponents: A New Way to Predict Chaotic Systems, in World Scientific Eds., Topics on Chaotic Systems: Selected papers from CHAOS 2008 International Conference: 158-165.

[14] Guégan, D. \& Mercier, L. (1998). Stochastic and chaotic dynamics in high-frequency financial data, in Prochazka Eds, Signal Processing and Prediction, 365-372.

[15] Lorenz, E.N. (1963). Deterministic non-periodic flow. Journal of Atmospheric Science, 20: 130-141.

[16] Murray, D. (1993). Forecasting a chaotic time series using an improved metric for embedding space, Physica D, 68: 318-325. 
[17] Shintani, M. \& Linton, O. (2004) Nonparametric neural network estimation of Lyapunov exponents and a direct test for chaos, Journal of Econometrics, 120: 1-33.

[18] Takens, F. (1996). Estimation of dimension and order of time series. Progress in Nonlinear Differential Equations and their Applications, 19: 405-422.

[19] Wolff, R.C.L. (1992). Local Lyapunov exponents: looking closely at chaos. Journal of the Royal Statistical Society B, 54: 353 - 371.

[20] Ziehmann, C., Smith, L.A. \& Kurths, J. (2000) Localized Lyapunov exponents and the prediction of predictability. Physics Letters A, 271: 237-251. 


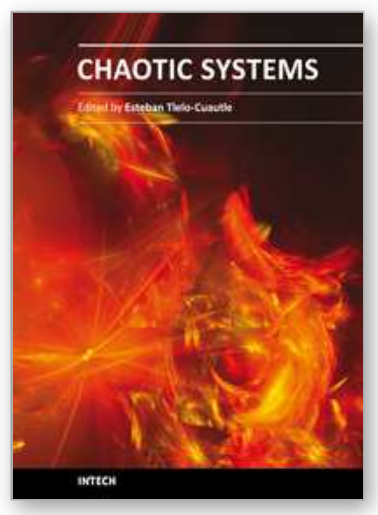

\author{
Chaotic Systems \\ Edited by Prof. Esteban Tlelo-Cuautle
}

ISBN 978-953-307-564-8

Hard cover, 310 pages

Publisher InTech

Published online 14, February, 2011

Published in print edition February, 2011

This book presents a collection of major developments in chaos systems covering aspects on chaotic behavioral modeling and simulation, control and synchronization of chaos systems, and applications like secure communications. It is a good source to acquire recent knowledge and ideas for future research on chaos systems and to develop experiments applied to real life problems. That way, this book is very interesting for students, academia and industry since the collected chapters provide a rich cocktail while balancing theory and applications.

\title{
How to reference
}

In order to correctly reference this scholarly work, feel free to copy and paste the following:

Dominique Guégan and Justin Leroux (2011). Predicting Chaos with Lyapunov Exponents: Zero Plays no Role in Forecasting Chaotic Systems, Chaotic Systems, Prof. Esteban Tlelo-Cuautle (Ed.), ISBN: 978-953-307-5648, InTech, Available from: http://www.intechopen.com/books/chaotic-systems/predicting-chaos-with-lyapunovexponents-zero-plays-no-role-in-forecasting-chaotic-systems

\section{INTECH}

open science | open minds

\section{InTech Europe}

University Campus STeP Ri

Slavka Krautzeka 83/A

51000 Rijeka, Croatia

Phone: +385 (51) 770447

Fax: +385 (51) 686166

www.intechopen.com

\section{InTech China}

Unit 405, Office Block, Hotel Equatorial Shanghai

No.65, Yan An Road (West), Shanghai, 200040, China

中国上海市延安西路65号上海国际贵都大饭店办公楼 405 单元

Phone: +86-21-62489820

Fax: +86-21-62489821 
(C) 2011 The Author(s). Licensee IntechOpen. This chapter is distributed under the terms of the Creative Commons Attribution-NonCommercialShareAlike-3.0 License, which permits use, distribution and reproduction for non-commercial purposes, provided the original is properly cited and derivative works building on this content are distributed under the same license. 